\title{
Relação da qualidade de vida com dados sociodemográficos e nutricionais de gestantes e puérperas
}

\section{Relationship between quality of life and sociodemographic and nutritional data in pregnant and postpartum women}

\section{Relación entre la calidad de vida y datos sociodemográficos y nutricionales de embarazadas y puérperas}

\author{
Liliane Maria Schumacher (iD \\ Universidade do Vale do Taquari - Lajeado - (RS) - Brasil \\ Mariane Schmitt iD \\ Universidade do Vale do Taquari - Lajeado - (RS) - Brasil \\ Patrícia Fassina \\ Universidade do Vale do Taquari - Lajeado - (RS) - Brasil \\ Fernanda Scherer Adami iD \\ Universidade do Vale do Taquari - Lajeado - (RS) - Brasil
}

\section{RESUMO}

Objetivo: Investigar a percepção da qualidade de vida de gestantes e puérperas e sua relação com dados sociodemográficos e nutricionais. Métodos: Estudo descritivo e transversal, de abordagem quantitativa, realizado em uma Estratégia de Saúde da Família do município de Lajeado, Rio Grande do Sul, no período de junho a setembro de 2019, com 57 gestantes e puérperas, submetidas a um questionário estruturado contendo dados sociodemográficos e clínicos e ao questionário WHOQOL-BREF. Avaliou-se o estado nutricional pré-gestacional e o ganho de peso para a idade gestacional, sendo os resultados considerados significativos a um nível de significância de $5 \%(p \leq 0,05)$. Resultados: 41 gestantes e puérperas consideraram sua qualidade de vida "boa" (72\%), 43 estavam "satisfeitas" ou "muito satisfeitas" com a sua saúde $(75,4 \%)$ e 32 apresentavam-se eutróficas $(56,1 \%)$. Verificou-se correlação inversa da avaliação da qualidade de vida com a idade $(p \leq 0,01)$ e o índice de massa corporal pré-gestacional esteve inversamente relacionado com a satisfação com a saúde $(p \leq 0,01)$ das gestantes e puérperas. As mulheres eutróficas apresentaram-se significativamente associadas à percepção de "muito satisfeita" em relação a sua saúde e aquelas com sobrepeso, à percepção de "insatisfeita" com a sua saúde $(p \leq 0,01)$. Conclusão: A avaliação da percepção da qualidade de vida associou-se inversamente à idade, assim como a percepção da satisfação com a saúde associou-se inversamente ao índice de massa corporal prégestacional das gestantes e puérperas investigadas.

Descritores: Qualidade de Vida; Gravidez; Período Pós-Parto.

\section{ABSTRACT}

Objective: To assess pregnant and postpartum women's perception regarding quality of life and its relationship to sociodemographic and nutritional data. Methods: This quantitative descriptive cross-sectional study was carried out in a Family Health Strategy center in the city of Lajeado - Rio Grande do Sul, from June to September 2019 with 57 pregnant and postpartum women who answered a structured questionnaire used to collect sociodemographic and clinical data and the WHOQOL-BREF questionnaire. Pregestational nutritional status and weight gain for gestational age were assessed, and the results were considered significant at a significance level of $5 \%$ ( $p \leq 0.05)$. Results: 41 pregnant and postpartum women rated their quality of life as good (72\%), 43 were either satisfied or very satisfied with their health (75.4\%) and were 32 were at normal weight (56.1\%). There was an inverse correlation between self-rated quality of life and age $(p \leq 0.01)$ and pregestational BMI was inversely related to satisfaction with health $(p \leq 0.01)$ in pregnant and postpartum women. Normal weight was significantly associated with being "very satisfied" with health, and overweight was associated with being "dissatisfied" with health ( $p \leq 0.01)$. Conclusion: Self-rated quality of life was 
inversely associated with age, and satisfaction with health was inversely associated with pregestational body mass index in the pregnant and postpartum women analyzed.

Descriptors: Quality of Life; Pregnancy; Postpartum Period.

\section{RESUMEN}

Objetivo: Investigar la percepción de calidad de vida de embarazadas y puérperas y su relación con datos sociodemográficos y nutricionales. Métodos: Estudio descriptivo y transversal de abordaje cuantitativo realizado en una Estrategia de Salud de la Familia del municipio de Lajeado, Río Grande de Sur Sul, en el período entre junio y septiembre de 2019 con 57 embarazadas y puérperas que contestaron una encuesta estructurada con datos sociodemográficos y clínicos y el cuestionario WHOQOLBREF. Se evaluó el estado nutricional antes del embarazo y la ganancia de peso para la edad gestacional y los resultados han sido considerados significativos con un nivel de significancia del $5 \%(p \leq 0,05)$. Resultados: 41 embarazadas y puérperas han considerado su calidad de vida "buena" (72\%), 43 estaban "satisfechas" o "muy satisfechas" con su salud (75,4\%) y 32 eran eutróficas (56,1\%). Se verificó una correlación inversa de la evaluación de la calidad de vida con la edad ( $p \leq 0,01)$ y el índice de masa corporal antes del embarazo estuvo inversamente relacionado con la satisfacción con la salud ( $p \leq 0,01)$ de las embarazadas y puérperas. Las mujeres eutróficas se presentaron significativamente asociadas con la percepción de "muy satisfecha" respecto su salud y aquellas con sobrepeso se asociaron con la percepción de "insatisfecha" con su salud (p $\leq 0,01)$. Conclusión: La evaluación de la percepción de calidad de vida se asoció de manera inversa con la edad así como la percepción de la satisfacción con la salud se asoció inversamente con el índice de masa corporal antes del embarazo y con las puérperas investigadas.

Descriptores: Calidad de Vida; Embarazo; Periodo Posparto.

\section{INTRODUÇÃO}

A gestação é um fenômeno fisiológico que envolve diversas mudanças e deve ser vista como parte de uma experiência de vida saudável ${ }^{(1)}$. Ela se desenvolve em um contexto social e cultural que influencia e determina a sua evolução e trajetória, sendo de grande importância considerar fatores como a história pessoal e a situação sociodemográfica e econômica da gestante(2), além dos fatores fisiológicos, psicológicos e sexuais que transpassam a vida das mulheres nesse período e que podem influenciar na qualidade de vida (QV) das mesmas ${ }^{(3)}$.

Conhecer o perfil das mulheres gestantes e identificar os determinantes de saúde que podem interferir no desenvolvimento saudável da gestação é essencial para que as equipes de saúde possam desenvolver ações de promoção que tragam a melhoria da qualidade de vida das gestantes ${ }^{(2)}$.

Assim como a gestação, o puerpério também é caracterizado por grandes oscilações físicas e emocionais que podem interferir na $Q V$ da puérpera( ${ }^{(4)}$. Poucas mulheres que estão vivenciando o pós-parto falam sobre suas inquietações e angústias, como dúvidas sobre seu peso, prevenção, medo da relação sexual, insegurança quanto ao contraceptivo, indisposições físicas e dificuldades emocionais ${ }^{(5)}$. Para uma melhor $Q V$ das puérperas, em relação ao seu autocuidado e ao do recém-nascido, muitas lacunas precisam ser esclarecidas ${ }^{(6)}$.

O conceito de QV é amplo, complexo, subjetivo, multidisciplinar e está relacionado a vários campos, não se prendendo a uma única definição ${ }^{(7)}$. Esse conceito tem se evidenciado como necessário na prática de cuidado dos diferentes grupos de pessoas após a ampliação do conceito de saúde, a qual passou a ser vista como o viver em sociedade acompanhado de bem-estar nos mais diversos sentidos e não apenas como a ausência de doença ${ }^{(8)}$.

Segundo a Organização Mundial da Saúde (OMS), a QV foi definida como a percepção do indivíduo de sua posição na vida no contexto da cultura e do sistema de valores nos quais ele vive e em relação aos seus objetivos, expectativas padrões e preocupações ${ }^{(9)}$, conceito que, apesar do passar do tempo, se mantém atual, e engloba a saúde física, psicológica, o nível de independência, as relações sociais, as crenças pessoais e a relação com o meio ambiente ${ }^{(10)}$.

A avaliação da QV tem sido um indicador utilizado para nortear práticas assistenciais e auxiliar na definição de estratégias de políticas públicas no âmbito de promoção de saúde e prevenção de doenças ${ }^{(11)}$, baseando-se no conceito ampliado de saúde para indivíduos e coletividades ${ }^{(12)}$. A promoção da saúde é composta por diversas estratégias que visam a atender as necessidades da população e garantir a melhoria da QV ${ }^{(13)}$.

As orientações da equipe de saúde precisam ir além das orientações na alta hospitalar e na visita domiciliar, sendo capazes de potencializar a forma de viver das mulheres nesse ciclo(6). É importante destacar a relevância dos serviços de saúde e do estabelecimento de vínculo entre a mulher e os profissionais de saúde diante do estado de fragilidade em que a mulher pode se encontrar no momento da chegada de um filho ${ }^{(14)}$. 
Nesse contexto, o presente estudo apresenta como objetivo investigar a percepção da qualidade de vida de gestantes e puérperas e sua relação com dados sociodemográficos e nutricionais.

\section{MÉTODOS}

O presente artigo trata-se de um estudo descritivo e transversal, de abordagem quantitativa, realizado no período de junho a setembro de 2019, em uma Estratégia de Saúde da Família (ESF) localizada município de Lajeado, no estado do Rio Grande do Sul, Brasil, com população estimada de 84.014 habitantes e Índice de Desenvolvimento Humano municipal de $0,778^{(15)}$.

A população do estudo constituiu-se por gestantes e puérperas que estavam em acompanhamento pré-natal na ESF no período da coleta de dados e que consentiram sua participação por meio da assinatura do Termo de Consentimento Livre e Esclarecido (TCLE). O convite para participar da pesquisa realizou-se no momento de aguardo das consultas com o profissional médico, enfermeiro, nutricionista, cirurgião-dentista, ou para algum grupo educativo da unidade. Considerou-se como critério de exclusão do estudo as gestantes e puérperas que desistiram de participar do estudo, mesmo após a assinatura do TCLE. Realizou-se a coleta de dados primários e secundários, sendo que a entrevista ocorreu em uma sala reservada da ESF.

Obteve-se o estado nutricional pré-gestacional das gestantes e puérperas por meio das medidas de peso pré-gestacional e estatura, coletados no prontuário da paciente, seguido pelo cálculo do Índice de Massa Corporal (IMC) (peso/altura ${ }^{2}$ ), com classificação segundo a World Health Organization (WHO), para adultas e para as adolescentes $^{(16,17)}$. Classificou-se o ganho de peso gestacional de acordo com a idade gestacional e o IMC prégravídico, através dos critérios do Institute of Medicine (IOM) ${ }^{(18)}$.

Também se utilizou o questionário WHOQOL-BREF para avaliar a QV das gestantes e puérperas, que é constituído por 26 questões. As duas primeiras perguntas avaliam a QV geral, tratando da percepção sobre sua QV e da satisfação com a saúde, e as 24 questões restantes representam cada uma das 24 facetas que compõem o instrumento original (WHOQOL-100), divididas em quatro domínios: físico, psicológico, relações sociais e meio ambiente. As respostas seguem uma escala de Likert (de 1 a 5 ; quanto maior a pontuação, melhor a qualidade de vida) ${ }^{(9)}$.

Além disso, aplicou-se um questionário estruturado com questões sobre dados sociodemográficos (idade, estado civil, escolaridade, ocupação da mãe, renda, número de pessoas que vivem na casa, tipo de moradia) e dados clínicos associados à gestação e ao puerpério (peso pré-gestacional, altura, número de filhos, ganho de peso na gestação e semana gestacional das gestantes e puérperas).

Adotou-se o software Statistical Package for the Social Sciences (SPSS), versão 22.0, e analisaram-se os dados através de tabelas estatísticas descritivas com a utilização dos testes estatísticos de associação, como o exato de Fischer, análise de correlação de Pearson, análise de correlação de Spearman e teste não-paramétrico de MannWhitney, e considerou-se um nível máximo de significância de $5 \%(p \leq 0,05)$.

O estudo recebeu aprovação do Comitê de Ética em Pesquisa da Universidade do Vale do Taquari sob o Parecer n. ${ }^{\circ} 3.336 .144$.

\section{RESULTADOS}

Observou-se, entre as gestantes e puérperas, a média de idade de 28,3 ( $\pm 6,6$ anos), um número médio de pessoas que vivem na casa de 2,9 ( $\pm 0,9$ pessoas) e a média do número de filhos anteriores de $0,7( \pm 0,9)$. Verificou-se a maior média de escore na avaliação da QV das gestantes e puérperas no domínio psíquico $(77,6 ; \pm 10,0)$, seguido do domínio social $(75,1 ; \pm 17,1)$, domínio ambiental $(63,5 ; \pm 10,5)$ e domínio físico $(62,6 ; \pm 12,1)$.

Participaram do estudo $75,4 \%(n=43)$ de gestantes e $24,6 \%(n=14)$ de puérperas, a maioria delas com idade entre 18 e 34 anos $(78,9 \% ; n=45)$, trabalhando fora de casa $(71,9 \% ; n=41)$ e vivendo em moradia própria $(77,2 \%$; $n=44)$, todas com companheiro. Em relação ao estado nutricional pré-gestacional, $56,1 \%(n=32)$ estavam eutróficas e $26,3 \%(n=15)$ com obesidade. Do total, $43,8 \%(n=25)$ apresentaram um ganho de peso acima do recomendado para a idade gestacional. A maioria das gestantes e puérperas considerou sua QV boa $(72 \% ; n=41)$ e estavam "satisfeitas" ou "muito satisfeitas" com a sua saúde (75,4\%; n=43), como apresenta a Tabela I.

A Tabela II apresenta que o ganho de peso adequado durante a gestação esteve significativamente associado às mulheres eutróficas, já o ganho de peso acima do recomendado esteve associado às gestantes com sobrepeso $(p=0,023)$. O teste de associação exato de Fisher demonstrou que as mulheres eutróficas apresentaram-se significativamente associadas à percepção de "muito satisfeitas" em relação à sua saúde e aquelas com sobrepeso, à percepção de "insatisfeitas" com a sua saúde $(p \leq 0,01)$. 
Tabela I - Descrição das características sociodemográficas das gestantes e puérperas. Lajeado, Rio Grande do Sul, Brasil, 2019.

\begin{tabular}{|c|c|c|c|}
\hline Variável & Resposta & $\mathbf{n}$ & $\%$ \\
\hline \multirow[t]{2}{*}{ Faixa de idade } & $18-34$ & 45 & 78,9 \\
\hline & 35 ou mais & 12 & 21,1 \\
\hline \multirow[t]{2}{*}{ Estado civil } & Com companheiro & 57 & 100,0 \\
\hline & Sem companheiro & - & - \\
\hline \multirow[t]{7}{*}{ Escolaridade } & Analfabeta & 1 & 1,8 \\
\hline & Ensino fundamental incompleto & 8 & 14,0 \\
\hline & Ensino fundamental completo & 9 & 15,8 \\
\hline & Ensino médio incompleto & 9 & 15,8 \\
\hline & Ensino médio completo & 21 & 36,8 \\
\hline & Ensino superior incompleto & 5 & 8,8 \\
\hline & Ensino superior completo & 4 & 7,0 \\
\hline \multirow[t]{2}{*}{ Ocupação } & Do lar & 16 & 28,1 \\
\hline & Trabalha fora de casa & 41 & 71,9 \\
\hline \multirow[t]{2}{*}{ Moradia } & Própria & 44 & 77,2 \\
\hline & Alugada & 13 & 22,8 \\
\hline \multirow[t]{4}{*}{ Estado nutricional pré-gestacional } & Magreza acentuada/Magreza & 2 & 3,5 \\
\hline & Eutrofia & 32 & 56,1 \\
\hline & Sobrepeso & 8 & 14,1 \\
\hline & Obesidade/Obesidade grave & 15 & 26,3 \\
\hline \multirow[t]{3}{*}{ Ganho de peso para a idade gestacional } & Abaixo do recomendado & 16 & 28,1 \\
\hline & Adequado & 16 & 28,1 \\
\hline & Acima do recomendado & 25 & 43,8 \\
\hline \multirow[t]{3}{*}{ Como você avaliaria sua qualidade de vida? } & Nem ruim nem boa & 8 & 14,0 \\
\hline & Boa & 41 & 72 \\
\hline & Muito boa & 8 & 14,0 \\
\hline \multirow{4}{*}{$\begin{array}{l}\text { Quão satisfeito(a) você está com a sua } \\
\text { saúde? }\end{array}$} & Insatisfeita & 3 & 5,3 \\
\hline & Nem satisfeita, nem insatisfeita & 11 & 19,3 \\
\hline & Satisfeita & 32 & 56,1 \\
\hline & Muito satisfeita & 11 & 19,3 \\
\hline
\end{tabular}

\%: percentual; n: número amostral 
Tabela II - Associação entre estado nutricional de eutrofia, sobrepeso e obesidade com a faixa etária, escolaridade, tipo de moradia, classificação do ganho de peso para a idade gestacional e percepção da qualidade de vida das gestantes e puérperas. Lajeado, Rio Grande do Sul, Brasil, 2019.

\begin{tabular}{|c|c|c|c|c|c|c|c|c|}
\hline \multirow[b]{3}{*}{ Variável } & \multirow[b]{3}{*}{ Resposta } & \multicolumn{6}{|c|}{ Estado nutricional } & \multirow[b]{3}{*}{$\mathbf{p}$} \\
\hline & & \multicolumn{2}{|c|}{ Eutrofia } & \multicolumn{2}{|c|}{ Sobrepeso } & \multicolumn{2}{|c|}{$\begin{array}{c}\text { Obesidade/ } \\
\text { Obesidade grave }\end{array}$} & \\
\hline & & $\mathbf{n}$ & $\%$ & $\mathbf{n}$ & $\%$ & $\mathbf{n}$ & $\%$ & \\
\hline \multirow[t]{2}{*}{ Faixa de idade } & $18-34$ & 26 & $81,3 \%$ & 5 & $62,5 \%$ & 12 & $80,0 \%$ & 0,528 \\
\hline & 35 ou mais & 6 & $18,8 \%$ & 3 & $37,5 \%$ & 3 & $20,0 \%$ & \\
\hline \multirow[t]{3}{*}{ Escolaridade } & Ensino fundamental & 9 & $28,1 \%$ & 3 & $37,5 \%$ & 5 & $35,7 \%$ & 0,917 \\
\hline & Ensino médio & 18 & $56,3 \%$ & 4 & $50,0 \%$ & 6 & $42,9 \%$ & \\
\hline & Ensino superior & 5 & $15,6 \%$ & 1 & $12,5 \%$ & 3 & $21,4 \%$ & \\
\hline \multirow[t]{2}{*}{ Moradia } & Própria & 22 & $68,8 \%$ & 7 & $87,5 \%$ & 14 & $93,3 \%$ & 0,157 \\
\hline & Alugada & 10 & $31,3 \%$ & 1 & $12,5 \%$ & 1 & $6,7 \%$ & \\
\hline \multirow{3}{*}{$\begin{array}{l}\text { Ganho de peso para a } \\
\text { idade gestacional }\end{array}$} & Abaixo do recomendado & 9 & $28,1 \%$ & 1 & $12,5 \%$ & 5 & $33,3 \%$ & 0,023 \\
\hline & Adequado & 14 & $43,8 \%$ & 1 & $12,5 \%$ & 1 & $6,7 \%$ & \\
\hline & Acima do recomendado & 9 & $28,1 \%$ & 6 & $75,0 \%$ & 9 & $60,0 \%$ & \\
\hline \multirow{3}{*}{$\begin{array}{l}\text { Como você avaliaria } \\
\text { sua qualidade de vida? }\end{array}$} & Nem ruim, nem boa & 4 & $12,5 \%$ & 1 & $12,5 \%$ & 3 & $20,0 \%$ & 0,981 \\
\hline & Boa & 23 & $71,9 \%$ & 6 & $75,0 \%$ & 10 & $66,7 \%$ & \\
\hline & Muito boa & 5 & $15,6 \%$ & 1 & $12,5 \%$ & 2 & $13,3 \%$ & \\
\hline \multirow{4}{*}{$\begin{array}{l}\text { Quão satisfeito(a) você } \\
\text { está com a sua saúde? }\end{array}$} & Insatisfeita & - & - & 2 & $25,0 \%$ & 1 & $6,7 \%$ & $\leq 0,01$ \\
\hline & Nem satisfeita, nem insatisfeita & 3 & $9,4 \%$ & 2 & $25,0 \%$ & 5 & $33,3 \%$ & \\
\hline & Satisfeita & 18 & $56,3 \%$ & 4 & $50,0 \%$ & 9 & $60,0 \%$ & \\
\hline & Muito satisfeita & 11 & $34,4 \%$ & - & - & - & - & \\
\hline
\end{tabular}

\%: percentual; n: número amostral; teste de associação exato de Fisher. Dessa análise foram excluídas da amostra duas mulheres com estado nutricional pré-gestacional de desnutrição e uma que declarou ser analfabeta

Na Tabela III observou-se que todas as correlações foram fracas, a partir da análise de correlação de Pearson. Apesar disso, encontrou-se significância estatística para algumas variáveis. Observou-se que, quanto maior 0 número de pessoas que vivem na casa $(p \leq 0,01)$ e maior o IMC prégestacional $(p=0,047)$, menor tende a ser a média do domínio físico. Quanto maior o número de pessoas que vivem na casa $(p=0,044)$ e maior o número de filhos anteriores $(p=0,020)$, menor tende a ser a média do domínio psíquico. Além disso, quanto maior o número de filhos anteriores $(p=0,020)$, menor tende a ser a média do domínio ambiental.

$\mathrm{Na}$ Tabela IV verificou-se correlação inversa da avaliação da qualidade de vida das gestantes e puérperas com a idade $(p \leq 0,01)$ e o IMC prégestacional esteve inversamente relacionado à satisfação com a saúde $(p \leq 0,01)$, utilizando-se a análise de correlação de Spearman.

Quando comparadas as médias dos domínios de QV entre as gestantes e puérperas, não se observou associação significativa, de acordo com o demonstrado pelo teste não paramétrico Mann-Whitney, porém as médias dos escores das gestantes, em todos os domínios, apresentaram-se superiores às das puérperas. O domínio psíquico apresentou as maiores médias e o domínio físico as menores médias entre as gestantes e puérperas, conforme dispõe a Tabela V. 
Tabela III - Correlação dos escores dos domínios de qualidade de vida com idade, renda familiar, número de pessoas que vivem na casa, índice de massa corporal pré-gestacional e número de filhos anteriores das gestantes e puérperas. Lajeado, Rio Grande do Sul, Brasil, 2019.

\begin{tabular}{lccccccccc}
\hline & \multicolumn{7}{c}{ Domínios da qualidade de vida } \\
\cline { 2 - 9 } Variável & \multicolumn{2}{c}{ Físico } & \multicolumn{2}{c}{ Psíquico } & \multicolumn{2}{c}{ Social } & \multicolumn{2}{c}{ Ambiental } \\
\cline { 2 - 9 } & $\mathbf{R}$ & $\mathbf{p}$ & $\mathbf{R}$ & $\mathbf{p}$ & $\mathbf{R}$ & $\mathbf{p}$ & $\mathbf{R}$ & $\mathbf{p}$ \\
\hline Idade & $-0,056$ & 0,680 & $-0,054$ & 0,692 & $-0,024$ & 0,859 & $-0,010$ & 0,939 \\
Renda familiar & $-0,124$ & 0,358 & 0,032 & 0,811 & 0,145 & 0,281 & 0,213 & 0,111 \\
Número de pessoas vivem na casa & $-0,366$ & $\leq 0,01$ & $-0,268$ & 0,044 & $-0,086$ & 0,526 & $-0,145$ & 0,282 \\
IMC pré-gestacional & $-0,265$ & 0,047 & 0,053 & 0,695 & $-0,107$ & 0,428 & $-0,216$ & 0,107 \\
Número de filhos anteriores & $-0,233$ & 0,081 & $-0,307$ & 0,020 & $-0,127$ & 0,348 & $-0,307$ & 0,020
\end{tabular}

Análise de correlação de Pearson; IMC: índice de massa corporal; R: relação; p: nível de significância

Tabela IV - Associação da percepção da qualidade de vida e satisfação com saúde, idade, renda, número de pessoas que vivem na casa, índice de massa corporal prégestacional e número de filhos anteriores das gestantes e puérperas. Lajeado, Rio Grande do Sul, Brasil, 2019.

\begin{tabular}{|c|c|c|c|c|}
\hline \multirow{3}{*}{ Variável } & \multicolumn{4}{|c|}{ Questões } \\
\hline & \multicolumn{2}{|c|}{$\begin{array}{c}\text { Como você avaliaria sua } \\
\text { qualidade de vida? }\end{array}$} & \multicolumn{2}{|c|}{$\begin{array}{l}\text { Quão satisfeita você está com a } \\
\text { sua saúde? }\end{array}$} \\
\hline & $\mathbf{R}$ & $\mathbf{p}$ & $\mathbf{R}$ & $\mathbf{p}$ \\
\hline Idade & $-0,350$ & $\leq 0,01$ & $-0,138$ & 0,307 \\
\hline Renda familiar & 0,114 & 0,396 & 0,118 & 0,384 \\
\hline Número de pessoas que vivem na casa & 0,000 & 1,000 & $-0,169$ & 0,210 \\
\hline IMC prégestacional & $-0,122$ & 0,367 & $-0,376$ & $\leq 0,01$ \\
\hline Número de filhos anteriores & $-0,063$ & 0,640 & $-0,124$ & 0,357 \\
\hline
\end{tabular}

Análise de correlação de Spearman; IMC: índice de massa corporal; R: relação; p: nível de significância

Tabela $V$ - Médias dos escores dos domínios e percepção da qualidade de vida das gestantes e puérperas. Lajeado, Rio Grande do Sul, Brasil, 2019.

\begin{tabular}{lccccc}
\hline Domínios & Situação da mulher & $\mathbf{n}$ & Média & Desvio padrão & p \\
\hline Físico & Gestante & 43 & 63,8 & 11,9 & 0,202 \\
Psíquico & Puérpera & 14 & 58,9 & 12,5 & 0,124 \\
& Gestante & 43 & 78,9 & 9,6 & 10,4 \\
Social & Puérpera & 14 & 73,5 & 17,2 & 0,055 \\
& Gestante & 43 & 77,3 & 15,7 & 0,351 \\
Ambiental & Puérpera & 14 & 68,5 & 10,8 & 0,6 \\
\end{tabular}

Teste não paramétrico Mann-Whitney; n: número amostral

\section{DISCUSSÃO}

O estado nutricional esperado para a população geral é a eutrofia, que, associada a uma alimentação saudável, é essencial para promover a saúde e prevenir doenças. Sabe-se que os estados nutricionais pré-gestacional e gestacional são importantes indicadores da evolução da gravidez, pois o desenvolvimento fetal é dependente do ambiente uterino que, por sua vez, se relaciona com uma ingestão de nutrientes adequada pela mãe $e^{(19)}$.

No presente estudo, verificou-se a predominância de gestantes com o estado nutricional pré-gestacional normal, assim como em estudo realizado com gestantes de um consultório obstétrico de Pato Branco, Paraná, em que 66,6\% 
das mulheres estavam eutróficas ${ }^{(20)}$, e em resultado encontrado em estudo realizado com 116 gestantes atendidas em um Centro de Saúde da Família situado no município de Sobral, Ceará, em que 45,5\% apresentavam-se com peso adequado ${ }^{(21)}$. No presente estudo, a maioria das gestantes e puérperas estudadas encontrou-se com o estado nutricional dentro do padrão esperado para a população geral, resultados que diferem do encontrado na pesquisa de Vigilância de Fatores de Risco e Proteção para Doenças Crônicas por Inquérito Telefônico (Vigitel), quando a frequência de eutrofia no conjunto da população brasileira perpassou $24,5 \%{ }^{(22)}$.

Observou-se, no presente estudo, uma alta taxa de mulheres que iniciaram sua gestação com sobrepeso/ obesidade, sendo $14 \%$ com sobrepeso e $26,3 \%$ com obesidade, totalizando $40,3 \%$ das mulheres com excesso de peso, corroborando estudo realizado em uma Unidade de Saúde da Família de Porto Velho, Rondônia, no qual 20,8\% das mulheres iniciaram sua gestação com sobrepeso e 20,9\% com obesidade, perfazendo $41,7 \%$ das mulheres com excesso de peso no início da gestação ${ }^{(23)}$, resultados inferiores aos constatados em pesquisa realizada em 26 capitais e o Distrito Federal com a população adulta, que demonstrou uma frequência de excesso de peso de $55,7 \%$ e de obesos de $19,8 \%^{(22)}$.

Já em outro estudo, realizado com 328 gestantes assistidas nas unidades de saúde da zona urbana de Vitória da Conquista, Bahia, 20,27\% das mulheres apresentaram-se com sobrepeso e 6,79\% com obesidade, totalizando $27,16 \%$ de excesso de peso pré-gestacional ${ }^{(24)}$. O rastreamento de gestantes com sobrepeso e obesidade é de grande importância, pois o baixo peso, o sobrepeso e a obesidade são fatores preocupantes para a mãe e para o bebêê(23).

Verificou-se, neste estudo, que $43,9 \%$ das mulheres apresentaram um ganho de peso acima do recomendado para a idade gestacional, estando significativamente associado às gestantes com sobrepeso no início da gestação, resultado semelhante ao encontrado em um estudo realizado com gestantes de Vitória da Conquista, Bahia, em que a prevalência de ganho de peso esteve em $42,5 \%$, com maior prevalência entre aquelas que iniciaram a gestação com sobrepeso/obesidade ${ }^{(24)}$.

Já em estudo realizado com gestantes assistidas na atenção básica de Cruzeiro do Sul, Acre, 59\% das gestantes avaliadas apresentaram ganho de peso excessivo, sendo predominante em todas as categorias de IMC pré-gestacional ${ }^{(25)}$. Visto que os estudos citados desenvolveram-se com gestantes de baixo risco, entende-se que medidas de prevenção do ganho de peso excessivo durante a gestação, como alimentação saudável e atividade física regular, são aliadas fundamentais no controle do peso, na redução do risco de doenças e na melhoria da $Q V^{(1)}$.

Considerando que a gestação é um período de risco para o desenvolvimento de obesidade e suas comorbidades em mulheres em idade fértil, ressalta-se a necessidade de monitoramento do ganho de peso e a realização de educação nutricional no prénatal, com atenção especial às mulheres de baixa renda e com sobrepeso/obesidade prégestacional(24). Segundo o IOM - Institute of Medicine Pregnancy Weight Guidelines ${ }^{(18)}$, a recomendação do ganho de peso durante a gestação deve ser de acordo com o estado nutricional inicial da gestante. Com base no IMC obtido na primeira consulta de pré-natal, é possível conhecer o estado nutricional atual e acompanhar o ganho de peso até o final da gestação. O ganho de peso excessivo durante a gestação e a falta de perda de peso após o parto são importantes indicadores de obesidade em longo prazo ${ }^{(26)}$.

Em relação à QV geral, no presente estudo, a maioria das gestantes e puérperas considerou sua QV "boa" e estava "satisfeita" ou "muito satisfeita" com a sua saúde, corroborando um estudo realizado com gestantes do município de Souza, Paraíba, no qual 81,67\% das gestantes considerou sua QV "boa" e 85,83\% estava "satisfeita" com a sua saúde ${ }^{(3)}$.

Em estudo realizado com gestantes de um município de Teresina, Piauí, na avaliação geral da satisfação sobre a qualidade de vida, $53,8 \%$ das gestantes considerou-se "insatisfeita"(27), constatando que a percepção sobre a saúde e a QV varia, inclusive, entre regiões geográficas próximas. Existem outros fatores que influenciam na percepção da saúde e da QV, como o comportamento alimentar, que caracteriza o modo como as pessoas se alimentam, considerando diversos aspectos da vida da pessoa, incluindo ambiente físico, social, psicológico, familiar, cultural, midiático e suas respostas comportamentais relacionadas ao ato de comer ${ }^{(28)}$.

Avaliar a qualidade de vida da gestante acompanhada na atenção primária à saúde é de grande importância para identificar se as ações realizadas estão contemplando as suas reais necessidades, além de fundamentar a criação de novas intervenções que contribuam para uma melhor qualidade de vida na gestação ${ }^{(27)}$.

As mulheres eutróficas deste estudo apresentaram-se significativamente associadas à percepção de "muito satisfeita" em relação a sua saúde e aquelas com sobrepeso, à percepção de "insatisfeita" com a sua saúde. A saúde passou a ser vista como o viver em sociedade, acompanhada de bem-estar nos mais diversos sentidos, e não apenas como a ausência de doença( ${ }^{(8)}$. É algo subjetivo e demarca situações materiais e universos culturais distintos nos estratos sociais, devendo-se ressaltar que o estudo das percepções das pessoas sobre a saúde pode prover um material importante para expor as condições de vida das mesmas, dando voz aos sujeitos ${ }^{(29)}$. 
Verificou-se, no presente estudo, a correlação inversa em relação à avaliação da QV com a idade, resultado diferente de um estudo realizado com grávidas de 35 anos ou mais, com baixa renda, em Natal, Rio Grande do Norte, que verificou que a idade mais avançada das gestantes pesquisadas pode ser vista como um fator que contribuiu positivamente para os escores de bem-estar subjetivo, podendo estar relacionada a uma maior maturidade ou com a experiência anterior de ser mãe ${ }^{(30)}$.

Já em estudo realizado em Encantado, Rio Grande do Sul, que comparou a QV de mulheres com idade até 59 anos, de 60 a 69 anos, e com 70 anos ou mais, concluiu-se que diferentes faixas etárias não apresentaram associação significativa em relação à percepção de $Q V^{(31)}$, assim como em estudo realizado em Belém, Pará, com mulheres do ambulatório de climatério, com idades entre 40 e 65 anos, que concluiu que o estado menopausa e a faixa etária não interferiram na QV das participantes do estudo ${ }^{(32)}$.

No presente estudo não se observou associação significativa entre as médias dos domínios de QV quando comparadas entre as gestantes e puérperas, porém as médias dos escores das gestantes, em todos os domínios, encontraram-se superiores às das puérperas. As limitações físicas causadas no puerpério imediato interferem na percepção da QV relacionada à saúde, estando também associadas ao número de consultas pré-natal e ao tipo de parto, o que reforça a importância de programas que possam amenizar os possíveis desconfortos no período pós-parto( ${ }^{(33)}$.

O planejamento e a prestação de uma assistência qualificada, pautada em um acompanhamento interdisciplinar à saúde da mulher, da gestante e do neonato, devem ser avaliados frequentemente, a fim de que essa assistência não se torne fragmentada e para que reflita em uma melhor QV nesse período ${ }^{(34)}$.

Quanto aos domínios de QV do presente estudo, o domínio psíquico entre as gestantes e puérperas apresentou a maior média, resultado diferente do encontrado em estudo realizado apenas com gestantes no estado do Acre, que encontrou menores escores da QV no domínio psicológico e ambiental ${ }^{(35)}$. No estudo realizado com gestantes de uma ESF do município de Souza, Paraíba, o domínio psicológico obteve a segunda maior média percentual de satisfação das gestantes, o que também difere do presente estudo ${ }^{(3)}$. O domínio psicológico contempla os sentimentos positivos e negativos, memória/concentração, autoestima, percepção sobre sua imagem corporal e espiritualidade ${ }^{(9)}$.

No domínio físico, no atual estudo, obteve-se a menor média de escore dos domínios de QV entre as gestantes e puérperas, corroborando os valores encontrados em um estudo realizado apenas com gestantes de uma ESF na periferia de São Bernardo do Campo, São Paulo, no qual o escore do domínio físico foi de $57,65^{(36)}$, e em um estudo realizado com gestantes de uma unidade de saúde de Cabo Frio, Rio de Janeiro, no qual a menor pontuação foi no domínio físico ${ }^{(37)}$. Esses resultados se justificam pela gestação e o puerpério acarretarem em uma menor satisfação em relação à dor e ao desconforto, dependência de medicação, energia e fadiga, mobilidade, sono e repouso, atividades de vida cotidiana e capacidade para o trabalho ${ }^{(3)}$.

Uma melhor QV da gestante está associada a um maior desempenho físico e menor número de desconfortos sentidos ao longo da gravidez ${ }^{(38)}$. No puerpério, a presença da dor e das limitações causadas por ela no pós-parto interferem na QV da puérpera e, na maioria das vezes, é pouco valorizada pelos profissionais de saúde, que negligenciam os cuidados maternos em detrimento dos cuidados neonatais ${ }^{(39)}$.

As limitações do presente estudo podem estar relacionadas à diferença entre o número de gestantes e puérperas que aceitaram participar, uma vez que as puérperas acessam menos a unidade, o que pode ter impactado na menor representatividade das puérperas no resultado.

Tendo em vista que a Política Nacional de Promoção da Saúde (PNPS) apresenta como objetivo promover a igualdade e a melhoria das condições e dos modos de viver, aumentando o potencial da saúde individual e coletiva e reduzindo vulnerabilidades e riscos à saúde ${ }^{(4)}$, entende-se que os resultados apresentados neste estudo podem contribuir no planejamento de ações de enfrentamento dos problemas relacionados à gestação e ao puerpério.

\section{CONCLUSÃO}

A avaliação da percepção da qualidade de vida associou-se inversamente à idade, assim como a percepção da satisfação com a saúde associou-se inversamente ao índice de massa corporal pré-gestacional das gestantes e puérperas investigadas.

\section{CONFLITOS DE INTERESSE}

Os autores declaram a inexistência de conflitos de interesses. 


\section{CONTRIBUIÇÕES}

Fernanda Scherer Adami, Liliane Maria Schumacher e Mariane Schmitt contribuíram com a elaboração e delineamento do estudo; a aquisição, análise e interpretação de dados; e a redação e/ou revisão do manuscrito. Patricia Fassina contribuiu com a redação e/ou revisão do manuscrito.

\section{REFERÊNCIAS}

1. Ministério da Saúde (BR), Secretaria de Atenção à Saúde, Departamento de Atenção Básica. Atenção ao prénatal de baixo risco [Internet]. Brasília: Ministério da Saúde; 2012 [acesso em 2020 Abr 20]. Disponível em: http://bvsms.saude.gov.br/bvs/publicacoes/cadernos_atencao_basica_32_prenatal.pdf

2. Rodrigues ARM, Dantas SLC, Pereira AMM, Silveira MAM, Rodrigues DP. Gravidez de alto risco: análise dos determinantes de saúde. Sanare [Internet]. 2017 [acesso em 2020 Abr 20];16(Supl 01):23-8. Disponível em: https://sanare.emnuvens.com.br/sanare/article/view/1135/620

3. Oliveira SX, Oliveira MB, Silva RAR, Davim RMB. Características dos domínios de qualidade de vida em gestantes da Estratégia de Saúde da Família. Rev Enferm UFPE [Internet]. 2013 [acesso em 2020 Abr 20];7(esp):7007-16. Disponível em: https://periodicos.ufpe.br/revistas/revistaenfermagem/article/ download/12370/15115\#: :text=O\%20dom\%C3\%ADnio\%20f\%C3\%ADsico\%20mostra\%20a,o\%20 trabalho\%2C $\% 20$ segundo $\% 20$ a\%20Fig

4. Chiarello V, Dias RG. Qualidade de vida do puerpério: possíveis relações com a prática de exercício físico na gestação. Corpo Ciênc Artes [Internet]. 2016 [acesso em 2020 Abr 20];6(1):24-38. Disponível em: http://www. ucs.br/etc/revistas/index.php/docorpo/article/viewFile/5357/2924

5. Corrêa MSM, Feliciano KVO, Pedrosa EM, Souza AI. Acolhimento no cuidado à saúde da mulher no puerpério. Cad Saúde Pública [Internet]. 2017 [acesso em 2020 Abr 20];33(3):e00136215. Disponível em: http://www.scielo.br/scielo.php?pid=S0102-311X2017000305011\&script=sci_abstract\&tlng=pt

6. Silva EC, Pereira ES, Santos WN, Silva RAR, Lopes NC, Figueiredo TAM, et al. Puerpério e assistência de enfermagem: percepção das mulheres. Rev Enferm UFPE [Internet]. 2017 [acesso em $2020 \mathrm{Abr}$ 20];11(Supl.7):2826-33. Disponível em: https://pdfs.semanticscholar.org/3b21/39c49a604fcaea1d4a57502be4 9aefd27abd.pdf

7. Cavalcante RMF, Pontes KV, Bandeira AA, Rocha AL. Qualidade de vida e qualidade de vida no trabalho: uma revisão bibliográfica. Id on Line Rev Mult Psic [Internet]. 2018 [acesso em 2020 Abr 20];12(40):21-31. Disponível em: https://idonline.emnuvens.com.br/id/article/view/882/1496

8. Rôla CVS, Silva SPC, Nicola PA. Instrumentos de avaliação da qualidade de vida de pessoas jovens e idosas: um estudo de revisão sistemática. Id on Line Rev Mult Psic [Internet]. 2018 [acesso em $2020 \mathrm{Abr}$ 20];12(42):111-20. Disponível em: https://idonline.emnuvens.com.br/id/article/view/1300/1892

9. The Whoqol Group. The World Health Organization quality of life assessment (WHOQOL): position paper from the World Health Organization. Soc Sci Med [Internet]. 1995 [acesso em 2020 Abr 20];41(10):1403-9. Disponível em: https://www.sciencedirect.com/science/article/abs/pii/027795369500112K?via\%3Dihub

10. Alcântara LAMB, Souza MAN, Almeida RJ. Aspectos da qualidade de vida de pessoas com lesão medular no Brasil: uma revisão integrativa. Saúde e Pesqui [Internet]. 2015 [acesso em 2020 Abr 20];8(3):569-75. Disponível em: https://www.researchgate.net/publication/289495750_Aspectos_da_Qualidade_de_Vida_de_ Pessoas_com_Lesao_Medular_no_Brasil_Uma_Revisao_Integrativa

11. Meneguin S, Xavier CL, Santana DG. Qualidade de vida de cardiopatas durante a gestação e após o parto. Acta Paul Enferm [Internet]. 2016 [acesso em 2020 Abr 20];29(2):232-38. Disponível em: http://www.scielo.br/ scielo.php?pid=S0103-21002016000200232\&script=sci_abstract\&tlng=pt

12. Dias MAS, Oliveira IP, Silva LMS, Vasconcelos MIO, Machado MFAS, Forte FDS, et al. Política Nacional de Promoção da Saúde: um estudo de avaliabilidade em uma região de saúde no Brasil. Ciênc Saúde Colet [Internet]. 2018 [acesso em 2020 Abr 20];23(1):103-14. Disponível em: http://www.scielo.br/scielo. php?pid=S1413-81232018000100103\&script=sci_abstract\&tlng=pt 
13. Malta DC, Morais NOL, Silva MMA, Rocha D, Castro AM, Reis AAC, et al. Política Nacional de Promoção da Saúde (PNPS): capítulos de uma caminhada ainda em construção. Ciênc Saúde Colet [Internet]. 2016 [acesso em 2020 Abr 20];21(6):1683-94. Disponível em: http://www.scielo.br/scielo.php?pid=S1413$81232016000601683 \&$ script $=$ sci_abstract\&tIng=pt

14. Zanatta E, Pereira CRR, Alves AP. A experiência da maternidade pela primeira vez: as mudanças vivenciadas no tornar-se mãe. Pesqui Prát Psicossociais [Internet]. 2018 [acesso em 2020 Abr 20];13(1):1-16. Disponível em: http://pepsic.bvsalud.org/scielo.php?script=sci_arttext\&pid=\$1809-89082018000100005

15. Instituto Brasileiro de Geografia e Estatística. História do município de Lajeado/RS [Internet]. 2017 [acesso em 2020 Abr 20]. Disponível em: https://cidades.ibge.gov.br/brasil/rs/lajeado/panorama

16. World Health Organization. Physical status: the use and interpretation of anthropometry [Internet]. 1995 [acesso em 2020 Abr 20]. Disponível em: https://apps.who.int/iris/bitstream/handle/10665/37003/WHO_ TRS_854.pdf;jsessionid=C2836FE4982D3E61EBC5EB32945655D0? sequence $=1$

17. World Health Organization. WHO child growth standards: lenght/height-for-age, weight-for-age, weight-forlength, weight-for-height and body mass index-for-age: methods and development [Internet]. 2006 [acesso em 2020 Abr 20]. Disponível em: https://www.who.int/childgrowth/standards/Technical_report.pdf?ua=1

18. Institute of Medicine (US), National Research Council (US), Committee to Reexamine IOM Pregnancy Weight Guidelines. Weight gain during pregnancy: reexamining the guidelines [Internet]. 2009 [acesso em $2020 \mathrm{Abr}$ 20]. Disponível em: https://www.ncbi.nlm.nih.gov/pubmed/20669500

19. Cunha LR, Pretto ADB, Bampi SR, Silva JMGC, Moreira AN. Avaliação do estado nutricional e do ganho de peso de gestantes atendidas em uma unidade básica de saúde de Pelotas-RS. Rev Bras Obes Nutr Emagrecimento [Internet]. 2016 [acesso em 2020 Abr 20];10(57):123-32. Disponível em: http://www.rbone. com.br/index.php/rbone/article/view/424

20. Demarchi AP, Freitas E, Baratto I. avaliação nutricional e laboratorial em um grupo de gestantes no Município de Pato Branco-PR. Rev Bras Obes Nutr Emagrecimento [Internet]. 2018 [acesso em 2020 Abr 20];12(72):466-78. Disponível em: http://www.rbone.com.br/index.php/rbone/article/view/726

21. Rufino MPR, Prado LS, Dias LT, Sousa JO, Frota MCQA, Carneiro JKR, et al. Avaliação do estado nutricional e do ganho de peso das gestantes atendidas em um Centro de Saúde da Família do interior norte do estado do Ceará/Brasil. Rev Interdisciplin [Internet]. 2018 [acesso em 2020 Abr 20];11(4):11-20. Disponível em: https://revistainterdisciplinar.uninovafapi.edu.br/index.php/revinter/article/view/1424/pdf_389

22. Ministério da Saúde (BR), Secretaria de Vigilância em Saúde, Departamento de Análise em Saúde e Vigilância de Doenças não Transmissíveis. Vigitel Brasil 2018: vigilância de fatores de risco e proteção para doenças crônicas por inquérito telefônico: estimativas sobre frequência e distribuição sociodemográfica de fatores de risco e proteção para doenças crônicas nas capitais dos 26 estados brasileiros e no Distrito Federal em 2018 [Internet]. Brasília: Ministério da Saúde; 2019 [acesso em 2020 Abr 20]. Disponível em: https://portalarquivos2.saude.gov.br/images/pdf/2019/julho/25/vigitel-brasil-2018.pdf

23. Alves KPS, Oliveira LML, Pedersoli AGA, Lemke GMMN. Estado nutricional e condições socioeconômicas em uma Unidade de Saúde da Família. Rev Saber Cient [Internet]. 2016 [acesso em 2020 Abr 20];5(1):61-8. Disponível em: http://revista.saolucas.edu.br/index.php/resc/article/view/587/pdf

24. Magalhães EIS, Maia DS, Bonfim CFA, Pereira M Netto MP, Lamounier JÁ, Rocha DS. Prevalência e fatores associados ao ganho de peso gestacional excessivo em unidades de saúde do sudoeste da Bahia. Rev Bras Epidemiol [Internet]. 2015 [acesso em 2020 Abr 20];18(4):858-69. Disponível em: http://www.scielo.br/scielo. php?script=sci_abstractmd=S1415-790X2015000400858\&Ing=en\&nrm=iso\&tlng=pt 25

25. Campos CAS, Malta MB, Neves PAR, Lourenço BH, Castro MC, Cardoso MA. Ganho de peso gestacional, estado nutricional e pressão arterial de gestantes. Rev Saúde Pública [Internet]. 2019 [acesso em 2020 Abr 20];53:57. Disponível em: http://www.scielo.br/scielo.php?pid=S0034-89102019000100248\&script=sci_ arttext\&tlng=pt

26. Associação Brasileira para o Estudo da obesidade e da Síndrome Metabólica. Diretrizes brasileiras de obesidade [Internet]. $4^{\mathrm{a}}$ ed. São Paulo: ABESO; 2016 [acesso em 2020 Abr 20]. Disponível em: https://abeso. org.br/wp-content/uploads/2019/12/Diretrizes-Download-Diretrizes-Brasileiras-de-Obesidade-2016.pdf 
27. Abreu K, Brandão A, Torres M. Qualidade de vida de gestantes acompanhadas na atenção primária à saúde. Saúde Redes [Internet]. 2019 [acesso em 2020 Abr 20];5(1):59-73. Disponível em: http://revista.redeunida. org.br/ojs/index.php/rede-unida/article/view/1697/PDF

28. Dantas RR, Silva GAP. O papel do ambiente obesogênico e dos estilos de vida parentais no comportamento alimentar infantil. Rev Paul Pediatr [Internet]. 2019 [acesso em 2020 Abr 20];37(3):363-71. Disponível em: http://www.scielo.br/scielo.php?pid=S0103-05822019005009101\&script=sci_arttext\&tlng=pt

29. Silva MJS, Schraiber LB, Mota A. O conceito de saúde na saúde coletiva: contribuições a partir da crítica social e histórica da produção científica. Physis [Internet]. 2019 [acesso em 2020 Abr 20];29(1):e290102. Disponível em: http://www.scielo.br/scielo.php?script=sci_arttext\&pid=S0103-73312019000100600\&lng=en\&n $\mathrm{rm}=$ iso\&tlng=pt

30. Oliveira MAM, Sousa WPS, Pimentel JDO, Santos KSL, Azevedo GD, Maia EMC. Gestantes tardias de baixa renda: dados sociodemográficos, gestacionais e bem-estar subjetivo. Psicol Teor Prát [Internet]. 2014 [acesso em 2020 Abr 20];16(3):69-82. Disponível em: http://pepsic.bvsalud.org/scielo.php?script=sci_arttext\& pid=S1516-36872014000300006

31. Lasta KC, Silva LG, Ferrari HR, Costa JLD, Dias CP, Tiggemann CL. Qualidade de vida de mulheres com diferentes faixas etárias de um programa de promoção à saúde. Rev Destaques Acad [Internet]. 2018 [acesso em 2020 Abr 20];10(3)36-45. Disponível em: http://www.univates.br/revistas/index.php/destaques/ article/view/1738/1392

32. Andrade RL, Fernandes ACM, Dias JRP, Laurindo BM, Vieira RC. Avaliação da qualidade de vida de mulheres climatéricas atendidas em ambulatório. Braz J Hea Rev [Internet]. 2019 [acesso em 2020 Abr 20];2(1):66-90. Disponível em: http://www.brjd.com.br/index.php/BJHR/article/view/847/727

33. Pedrosa NS, Cortês DB, Fernandes KC, Araújo MFS, Rocha APR, Carmo EM. Percepção da qualidade de vida no puerpério imediato. Colloquium Vitae [Internet]. 2013 [acesso em 2020 Abr 20];5(especial):209-15. Disponível em: https://pdfs.semanticscholar.org/cbc0/c453f7369e942cd4f888a64d2dc12f5a83eb.pdf

34. Castro GG, Ferreira FFG, Camargos AS, Leite MAFJ, Mattos JGS. Diferenças da qualidade de vida entre mulheres com alto e habitual risco gestacional. Aletheia [Internet]. 2019 [acesso em 2020 Abr 20];52(1):10215. Disponível em: http://www.periodicos.ulbra.br/index.php/aletheia/article/view/5292

35. Santos AB, Santos KEP, Monteiro GTR, Prado PR, Amaral TLM. Autoestima e qualidade de vida de uma séria de gestantes atendidas em uma Rede Pública de Saúde. Cogitare Enferm [Internet]. 2015 [acesso em 2020 Abr 20];20(2):392-400. Disponível em: https://revistas.ufpr.br/cogitare/article/view/38166/25540

36. Castro DFA, Fracolli LA. Qualidade de vida e promoção da saúde: em foco as gestantes. Mundo Saúde [Internet]. 2013 [acesso em 2020 Abr 20];37(2):159-65. Disponível em: http://www.saocamilo-sp.br/pdf/ mundo_saude/102/4.pdf

37. Lima ACN, Oliveira FB, Avolio GP, Silva GD, Silva PS, Vale RGS. Prevalence of low back pain and interference with quality of life of pregnant women. Rev Dor [Internet]. 2017 [acesso em 2020 Abr 20];18(2):119-23. Disponível em: http://www.scielo.br/scielo.php?script=sci_arttext\&pid $=$ S1806-00132017000200119

38. Afonso A, Quitério D, Sousa F, Sousa L, Silva R, Henriques CMG, et al. A influência dos factores demográficos, sociais, económicos, e obstétricos na qualidade de vida da grávida. Inter J Develop Educ Psychol [Internet]. 2011 [acesso em 2020 Abr 20];4(1):39-44. Disponível em: https://dialnet.unirioja.es/servlet/ articulo?codigo $=5575447$

39. Beleza ACS, Ferreira CHJ, Sousa L, Nakano AMS. Mensuração e caracterização da dor após episiotomia e sua relação com a limitação de atividades. Rev Bras Enferm [Internet]. 2012 [acesso em $2020 \mathrm{Abr}$ 20];65(2):264-8. Disponível em: http://www.scielo.br/scielo.php?pid=S0034-71672012000200010\&script=sci_ abstract\&tlng=pt

40. Ministério da Saúde (BR), Secretaria de Vigilância em Saúde, Secretaria de Atenção à Saúde. Política Nacional de Promoção da Saúde: PNPS: anexo I da Portaria de Consolidação $n^{\circ} 2$, de 28 de setembro de 2017, que consolida as normas sobre as políticas nacionais de saúde do SUS [Internet]. Brasília: Ministério da Saúde; 2018 [acesso em 2020 Abr 20]. Disponível em: http://bvsms.saude.gov.br/bvs/publicacoes/politica_ nacional_promocao_saude.pdf 
Endereço do primeiro autor:

Liliane Maria Schumacher

Universidade do Vale do Taquari

Rua Avelino Talini, 171

Bairro: Universitário

CEP: 95.914-014 - Lajeado - RS - Brasil

E-mail: lilischu@universo.univates.br

\section{Endereço para correspondência:}

Fernanda Scherer Adami

Universidade do Vale do Taquari

Rua Avelino Talini, 171

Bairro: Universitário

CEP: 95.914-014 - Lajeado - RS - Brasil

E-mail: fernandascherer@univates.br

Como citar: Schumacher LM, Schmitt M, Fassina P, Adami FS. Relação da qualidade de vida com dados sociodemográficos e nutricionais de gestantes e puérperas. Rev Bras Promoç Saúde. 2020;33:10571. 